\title{
Study on Teaching of English Reading: Under Thematic Progression Model
}

\author{
Zhanming Wang \\ College of International Studies, Southwest University, Chongqing, China \\ Email: zhan-ming-wang@hotmail.com
}

Received 21 January 2015; accepted 11 February 2015; published 13 February 2015

Copyright (C) 2015 by author and Scientific Research Publishing Inc.

This work is licensed under the Creative Commons Attribution International License (CC BY).

http://creativecommons.org/licenses/by/4.0/

c) (i) Open Access

\begin{abstract}
Thematic progression pattern is one of important ways to construct discourse. It can help readers understand the content of discourse and methods of how writer organize the information of the discourse profoundly by means of analyzing thematic progression. In this paper, we reviewed some thematic progression patterns raised by different scholars, analyzed and discussed thematic progression according to a model essay, and found out the significance and feasibility in teaching of English reading under thematic progression model, which was based on the explanation and illustration of thematic progression model.
\end{abstract}

\section{Keywords}

Theme, Rheme, Theme Progression, Teaching of English Teaching

\section{Introduction}

Discourse is a language unity which is made up of a series of correspondent successive sentence groups or is made up of groups of relevant sentences. If we want to write a successful discourse, we have to make the semantics coherent and conform to grammar. That is to say in a discourse, logical construction and scientific proposition should exist. The reason why a prominent literary work is fascinating is not only because of the writer's extraordinary arrangement of plot and content, but also the graceful and delicate language of this work. To find a proper language expression form for works is an ultimate state of many writers. Therefore, both content and form of discourse are the starting points to analyze literature works. That is because content is embodied by form, form is realization means of content, and form itself is always bearing meaning and literariness of discourse. There must be some semantic connections and logical links among sentences. On the basis of this kind of construction, paragraphs and sentences in discourse should be formed as formal connection and meaning 
links. That is cohesion and coherence of a discourse. When we cannot understand what the sentence is conveying, theme analysis could be used to understand the sentence. At the same time, thematic progressive model can help readers make a better understanding about writers' train of thought, meaning of the article and methods of organizing language to a certain degree. And it is an important method for discourse generation, which embodies textual meaning of this discourse, and reflects organizational methods of conceptual meaning and interpersonal meaning. It also provides writers with a method to explore the sentence combination and content relations. Thematic progression pattern is widely used for discourse analysis. Many scholars combine it with concrete discourse types to do further study, such as discussing how to use it in science, advertisement, news and medical science discourse. However, the combinations of thematic progression with literary works are not so many, just two examples which are Li Guoqing's study about the relation between thematic progression pattern with discourse genre about The Old Man and Sea, and Zhang Man's discussion about variation and coherence of thematic progression pattern in stream of consciousness novels. In this paper, we try to go a step further to study theme theory and analyze the theme of some articles to find out the significance and feasibility of thematic progression in teaching of English reading.

\section{Thematic Progression}

\subsection{Introduction}

In recent several decades, theme structure is always a hot topic of language study. As the development of the study of theme structure, the study range gradually expands from clause to discourse.

Thematic progression pattern theory was first proposed by Danes, 1974, which has more than thirty years' history. As an important constituent of discourse organization study, thematic progression pattern is influencing structure of style or manner of writing of discourse and coherence of the whole essay. To study the relationship of thematic progression can not only help to describe the language itself scientifically, but also do help to the development of other branches of study.

\subsection{Inquire into Basic Theory of Thematic Progression}

\subsubsection{Thematic Progression: From Clause to Discourse}

Halliday’s assumption (Halliday, 1994) about discourse function is in the level of semantics, which organizes language elements into discourse, and embodies through theme structure, information structure and cohesion. Theme structure theory is the core theory for the micro analyze of discourse in Halliday's systemic-functional grammar. In his theory, clause is an information spot, and theme is a target which is discussed, and is the starting point of the clause. Theme is located in front of the clause, while reheme is words revolving the clause. In the book An Introduction to Functional Grammar, theme structure is semantic structure of clause, therefore, Halliday always discussed segment of theme and rheme and the relationship of information structure in level of clause. However, this kind of study has its own limitation in discourse level (Hu, Zhu, Zhang, \& Li, 2005). In an independent sentence, theme and rheme are fixed. However, a discourse is made up of several sentences, while themes and rhemes of sentences are changing, connecting with form and meaning. The changing and connection between themes and rhemes is called progression. As every sentence progress forward, the whole discourse is unfolded, and the unity which can express a complete meaning is formed.

Danes put forward thematic progression in early times. He thought that theme structure of a discourse was the link and rank of theme, relation and level, relation of paragraph, the whole discourse and situation. He named all those complicated thematic relation in a discourse thematic progression (Danes, 1974). If theme embodies the specific information conveyed by speakers or discourse writers (including starting point of information, appointed experience meaning and interpersonal meaning), thematic progression pattern reveals the methods and ways of relevant information conveyed by speakers, or reveals writing thoughts and expressing intentions of discourse writers.

\subsubsection{Discussion about Thematic Progression Pattern}

After massive analysis on discourse, Danes put forward five types of thematic progression patterns, which is a) Simple Linear; b) TP with A Constant Theme; c) TP with Derived Themes; d) Split Rheme Pattern; e) Skipping 
Theme Pattern. The former three are comparatively common, which had influenced a lot about discourse analysis. After Danes' study, a lot of scholars from China and abroad had delved deeper to study thematic progression pattern, however, different scholars have different division about basic thematic pattern because of different corpus, ways of logical thinking and reference point.

$\mathrm{Xu}$ Shengheng and other Chinese linguists divided thematic patterns one after another. Zhu Yongsheng and Yan Shiqing concluded four types of patterns after studied other linguists' study (Zhu \& Yan, 2000), which is a) Theme Identity; b) Duration; c) Rheme Identity; d) Cross-Connection, corresponding with four types of Xu. Moreover, they called two or more than two theme patterns as one special pattern, namely, compound pattern. It should be noted that in language use, compound patterns are the most common ones. Discourse progresses according to one simple pattern exist, but not many. Most thematic progression of discourses are complicated. Because the complexity of thinking expression itself on one hand, rhetoric and expression purpose, reasonable arrangement of information, avoidance of monotony of forms should be considered on the other hand. So it is more common that several patterns go with each other and being used alternatively.

\subsection{Present Situation and Reflection of Thematic Progression Study}

In order to make an understanding about present situation of thematic progression study and prospect its development and application, we used China National Knowledge Infrastructure (CNKI) to retrieval papers about thematic progression from the year 1994 to 2006.

\subsubsection{Developing Trend of Quantity of Relevant Study Papers}

According to the outcome, there are 36 papers in total which are about thematic progression in Chinese core journals, while 203 papers are published in all kinds of journals. From 1980s, Xu Shengheng, Huang Yan and Fang Yan had published several papers about thematic progression, but not so many. Throughout 1980s till now, the number of papers about this field is increasing. The development can be divided roughly into two periods: initial stage, from 1980s to 1990s, is introduction of theme and rheme theory and progression theory; active stage, after 2000 till now, whose achievements are increasing, developing to applicative analysis, discovering and discussing application of thematic progression in translation, genre analysis and teaching.

\subsubsection{Content of Those Papers}

We have divided those 36 papers into 6 types, and among these, 13\% for translation application, 13\% for teaching application, $27 \%$ for discourse analysis, $13 \%$ for information structure, $27 \%$ for cohesion and $7 \%$ for all the other kinds of study.

We can further divide those 6 types into two kinds, which is theoretical study and applied analysis. The former is $40 \%$ while the latter is $53 \%$.

\section{1) Theoretical study}

Theoretical study including the study which connects thematic progression with cohesion, and information structure. Halliday's assumption about discourse function is embodies by three ways: theme structure, information structure and cohesion. A lot of papers are about discourse function in functional grammar to discuss connection of thematic progression with information structure and cohesion.

There are four papers are about the combination of thematic progression with information structure, which analysis the relation between thematic progression with information flow, and their links and functions in discourse. Ju Yumei (Ju, 2003) studied information structure of discourse combining with theme structure, thinking that thematic progression pattern reflected distribution of discourse information. Wang Qi (Wang \& Cheng, 2004) proposed that information system can solve the problem about formalization of thematic progression through endowing information parameter to theme. Liu Wencui and Cui Guihua (Liu \& Cui, 2006) compared features and relations about theme and rheme structure and information structure. There are 8 papers which are about relations between thematic progression with cohesion. They are focusing on how thematic progression functions its discourse function in discourse cohesion. Those papers discussed relations between thematic progression with information structure and cohesion. They are intent to import and introduce the theory and its function to China, and make a strong foundation for further developing and applying the theory.

\section{2) Application analysis}


Application analysis is built on theoretical study, turning to practical application, including discourse genre analysis, translation application and teaching application.

\section{1) Discourse genre analysis}

Fries (Fries, 1995) once inferred that different discourse genre would embody different features and trends of thematic progression, like thematic progressions of narration and exposition are different. For one discourse, different stages would also have different thematic progression. After Fries, a lot of Chinese scholars had discovered this field, so we have some papers about it.

Zhu Yongsheng (Zhu, 1995) once proposed that there were three functions of theme patter study: a) To reflect ways of topic development in one discourse and to reflect how different parts of a discourse connect with each other in semantics and logic; b) To compare same genre of discourse from the perspective of thematic progression; c) To find out the difference about selection of thematic progression about genre of discourse, namely, how genre influence adoption of theme pattern to discourse.

In China, there are 8 papers on discourse genre study using theme and thematic progression. Among these, one is about studying The Old Man and Sea (Li, 2003), while analysis about the same genre is heating. There are 6 papers are about studying the same genre, involving science, inaugural address, narration, argumentative essay, news interview and editorials discourse. The coverage is not wide, and it can be further expanded. By comparison, papers about comparing thematic progression of different genres of discourse are quite a few, needing us to find out. Meanwhile, on comparison of English and Chinese, there just one paper which is about the difference of argumentative essay of English and Chinese (Xu, 1995). In that paper, the writer admitted that when comparative analysis of cross-language extends to the level of discourse, connection of language use and culture is becoming more and more complicated; what kind of difference of the same genre in different cultures need to be further discussed. Up to now, the segment of theme and rheme in Chinese is due to a dispute, therefore, this topic need to be further improvement to do further discussion.

For study methods part, 8 papers all have imperfection about material selection, quantity of material being too small. Some papers chose paragraphs at random, which ignored the change of intention and purpose in every paragraph leading to the change of language in development of discourse structure. Therefore, whether those papers have proved that discourse genre can illustrate rule and feature of the selection of thematic progression or not. Those papers are good trials using thematic progression pattern to analyze discourse genre, which leave us more questions worth thinking and verifying.

\section{2) Translation application}

In translation field, people began to use theme structure theory for reference 20 years ago. Most of the researchers combined Prague School with Systemic-functional School to analyze information distribution at sentence level and cohesion at discourse level. In recent years, Chinese researchers began to pay attention to structure and cohesion at discourse level rather than word order at sentence level. There are 5 papers in core journal, trying to solve practice problems with theme and thematic progression pattern theory. Some writers analyzed translation strategy through thematic progression as inner condition of cohesion (Wang, 1998); some eliminated readability barrier of translated text from the perspective of thematic progression (Chen, 2002); some used discourse function of thematic progression to discuss deconstruction function of English-Chinese and ChineseEnglish translation (Wang, 2000); some discovered how theme and rheme theory function on automatic management of English abstract in computational linguistics (Deng, 2003); some discussed transforming rules of theme pattern between source and target language (Liu, 2006), proposing that if norms for thematic progression complies with target language, communicated effect generated by source information structure can be reproducted.

\section{3) Teaching application}

There are 4 papers about teaching application of thematic progression. The research method is to use discourse cohesion function of thematic progression and relation between thematic progression pattern with discourse genre feature, to discover the function of it in reading writing teaching. And they turn to example analysis to find out guiding meaning of thematic progression to teaching practice. Xiang Lan (Xiang, 2002) analyzed how to help students to use thematic progression to understand reading text from reading teaching perspective, grasping discourse content from top to bottom. Tan Xiaolan (Tan, 2006) discussed how teachers can help students to arrange thematic progression to construct discourse from the perspective of writing. 


\section{Thematic Progression and Teaching of English Reading}

The famous linguist from Czekh in his book about discourse construction once said that theme is quite worthy of narrating. We can see it clearly from sentence constituent. The reason why theme is the important means to construct discourse is it bears little information. Therefore, usually we can regard every discourse as different theme sequence. When the development of frame of discourse is planning by thematic structure of discourse, the first theme of the sentence is often the developing guiding principle or the topic of discourse. The remaining themes of this discourse basically progress around this guiding principal. If we rank theme of every sentence in an order we can make theme structure of discourse be seen clearly and explicitly. In this way readers can not only fully understand writer's train of thought, but also can shorten the time of reading this discourse. Let us analysis thematic progression pattern specifically and take the discourse I Was Later for the Train as an example. We choose this discourse because it is a compound pattern. In actual situation, compound pattern is the most frequently appeared one, rather than single pattern. However, discourse with compound pattern is usually elusive, we use schematic diagram to show how those themes progress and how to teach student to read such discourse.

1) The train I was travelling on was already half an hour late; 2) I had arranged to arrive in Vienna at 7:15 in time to catch the 7:25 train to Paris; 3) But there was no hope of that now; 4) I explained the situation to the ticket-collector who advised me to get off two stops before Vienna Central Station and take a taxi; 5) When the time came, he even helped me with my luggage; 6) He wished me good luck as I jumped off; 7) And a few minutes later I was racing towards the center of the city in taxi; 8) It was almost 7:25 when we stopped outside the station; 9) I paid the driver quickly, seized my bags and hurried inside; 10) "Paris train?" was all I had time to say to the first official I saw; 11) You can imagine my disappointment when he pointed to a train that was just moving out of the station at great speed.

This discourse is a narration, which is about how the writer did not catch the train. According to Image 1 we can see that the macro theme of this discourse is I (T2, T4, T7 \& T9), which is the content to push discourse to progress. If we regard macro theme "I" as dividing point of this discourse, we can divide it as three levels: the first three sentences are introduction ("I" would be late to catch the train to Paris); sentence four to eight are the major part of the whole discourse ("I" tried my best to catch Paris train); this part can be further divided into two parts: one is sentence four to six and the other is sentence seven to eight; the last three sentence are conclusion part (“i” missed the Paris train).

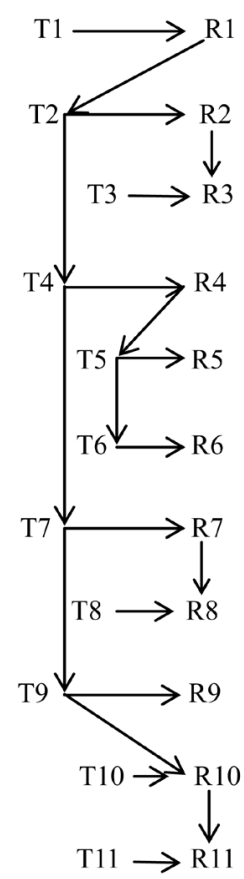

Image 1. Discourse I was late for the train. 


\section{Conclusion}

After analyzing this discourse, we can see that to analyze theme structure scientifically, in a manner of speaking, can help to analyze discourse further. On one hand, it can guide students to grasp writers' purpose of composing this discourse, train of thought and sequence; on the other hand, it can also help students to make a better understanding about information structure of discourse when they are learning discourse reading. That is because theme structure and information structure are not detached, on the contrary, they are highly connected. In most situations, we can understand theme structure as information structure. But what we have to know is that we can use all kinds of method to analyze discourse, and thematic progression pattern is just one of the patterns.

Besides, the process of interpretation towards discourse is just dynamic, not static. This process is also the communication and cooperation of writers and readers. It should not be conducted passively, but actively. Therefore when readers are reading papers, they should combine their culture and background knowledge to infer and examine of the discourse reasonably, to make a perfect understanding about it.

\section{References}

Chen, Z. (2002). Readability and Exclusion of Translation Text. China Translation, 3, 126-135.

Danes, F., Ed. (1974). Papers on Functional Sentence Perspective. The Hague: Mouton.

Deng, S. (2003). Discourse Translation Study: Based on Theme and Rheme Theory. Journal of Southwestern University for Nationalities, 24, 287-291.

Fries, P. H. (1995). Methods of Development, and Texts. On Subject and Theme-A Discourse Functional Perspective. Amsterdam: John Benjamins Publishing Co.

Halliday, M. A. K. (1994). An Introduction to Functional Grammar (2nd ed.). London: Edward Amold.

Hu, Z., Zhu, Y. S., Zhang, D. L., \& Li, Z. Z. (2005). Introduction of Systemic-Functional Grammar. Beijing: Peking University Press.

Ju, Y. M. (2003). Study on Informational Structure: From Perspective of Functional Linguistics. Foreign Language and Teaching, 4, 86-93.

Li, G. Q. (2003). Thematic Progression and Discourse Genre: Analysis of The Old Man and Sea. Foreign Language and Teaching, 7, 23-34.

Liu, F. L. (2006). Thematic Progression Pattern in English-Chinese Translation. Foreign Language Teaching and Research, 5, 45-52.

Liu, W. C., \& Cui, G. H. (2006). Comparison Study on Theme and Rheme Structure and Information Structure. Journal of Northeast Normal University, 6, 102-107.

Tan, X. L. (2006). To Improve Ability of Construction of English Discourse of Students-Problems about English Writing Teaching in University. Journal of Shanghai University (Social Science Edition), 2, 56-64.

Wang, B. (2000). Translation Deconstruction and Structure Function of Thematic Progression. China Translation, 1, 35-37.

Wang, D. F. (1998). Discourse Cohesion and Preliminary Study on Translation. Foreign Language and Teaching, 6.

Wang, Q., \& Cheng, X. T. (2004). Thematic Progression and Information Parameter in Discourse. Journal of Foreign Languages, 2, 48-52.

Xu, L. S. (1995). Theme Pattern and Discourse Analysis. Foreign Language Teaching and Research, 3, 6-12.

Xiang, L. (2002). Application of Reading Teaching of Thematic Progression. Foreign Language Teaching and Research, 3 , 23-24.

Zhu, Y. S., \& Yan, S. Q. (2000). Reflections on Systemic Functional Linguistics. Shanghai: Shanghai Foreign Language Teaching Press.

Zhu, Y. S. (1995). Theme Pattern and Discourse Analysis. Foreign Language Teaching and Research, 3, 6-12. 
Scientific Research Publishing (SCIRP) is one of the largest Open Access journal publishers. It is currently publishing more than 200 open access, online, peer-reviewed journals covering a wide range of academic disciplines. SCIRP serves the worldwide academic communities and contributes to the progress and application of science with its publication.

Other selected journals from SCIRP are listed as below. Submit your manuscript to us via either submit@scirp.org or Online Submission Portal.
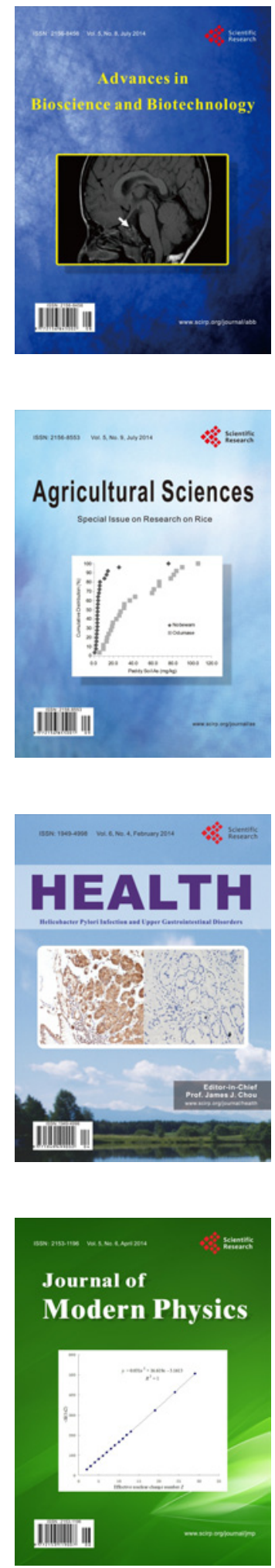
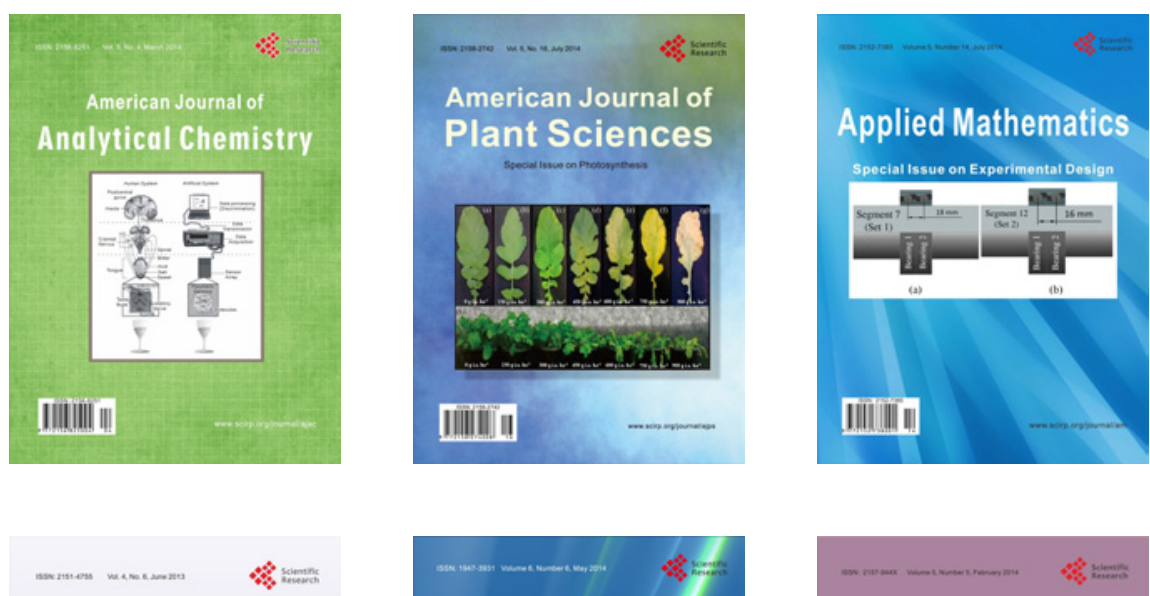

Creative Education
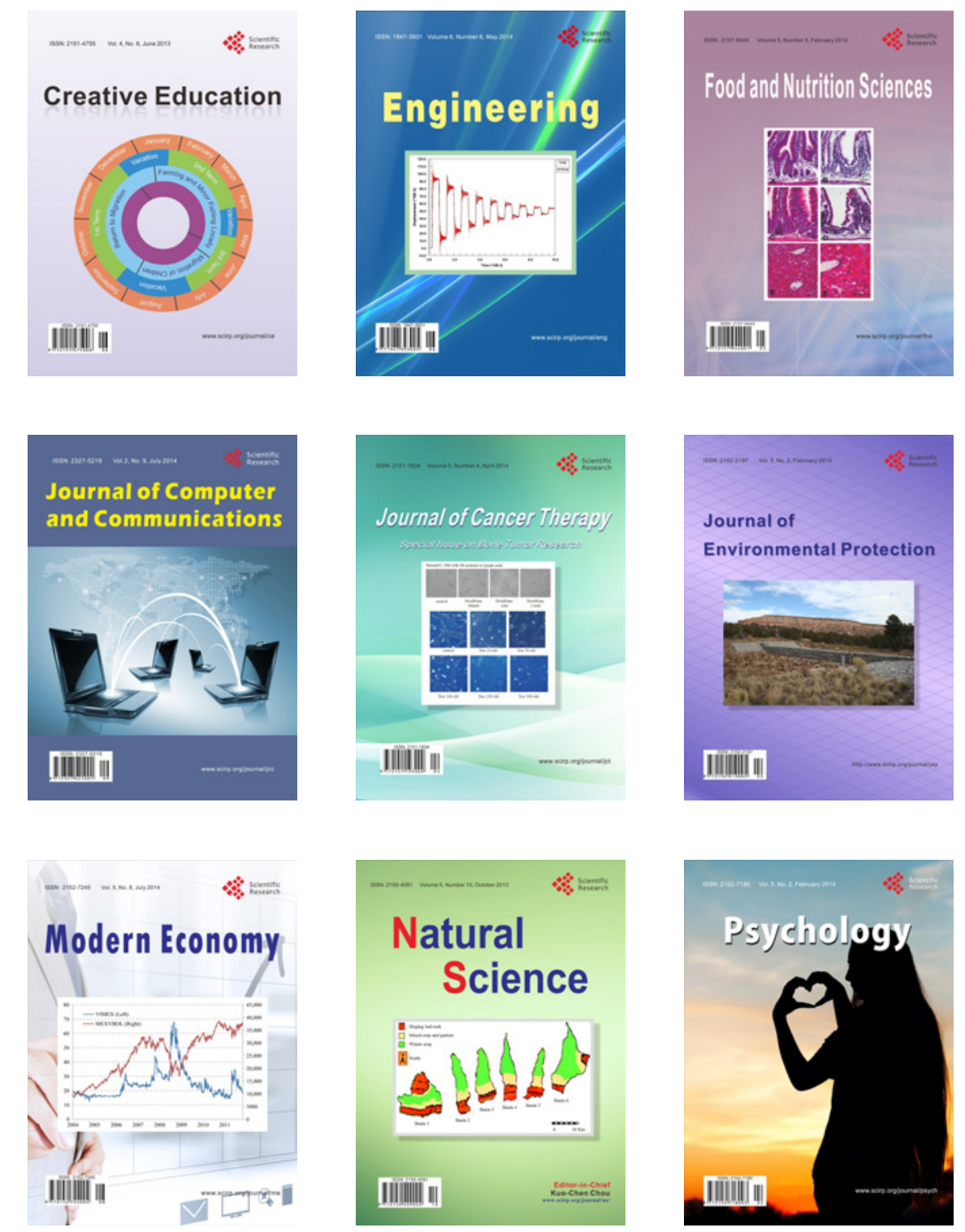